\title{
THE IMPACT OF INTRADIALYTIC EXERCISE ON ARTERIAL STIFFNESS AND ECHOCARDIOGRAPHIC PARAMETERS IN HEMODIALYSIS PATIENTS
}

Salah Said Ebraheem Naga*, Eman Salah Eldein Khaliel, Amira Hussein Mohamed, Amany Mohamed Seddik, Mona Saeed Abbas Ginidy

Department of Internal Medicine*, Faculty of Medicine, Alexandria University, and Department of Experimental and Clinical Internal Medicine, Medical Research Institute, Alexandria University.

\section{Introduction}

Cardiovascular disease (CVD) is the major cause of morbidity and mortality in patients with end-stage renal disease (ESRD) on haemodialysis (HD). The increased risks of cardiovascular events and mortality in dialysis patients is partly related to an increased prevalence of traditional cardiovascular risk factors, including diabetes mellitus, hypertension, obesity, physical inactivity, smoking and dyslipidaemia. However, these factors account for less than $50 \%$ of the excess risk of cardiovascular disease, leading many researchers to explore the roles of non-titionl risk factors, including anaemia chronic inflammetion, he the heightened carliovascular risk in ESRD, causing hemodynamic altertions via mithe pathays. Musce wasting, abormalities in muscle function, ald effects on execise perfors. Musce wang. a peformace are com

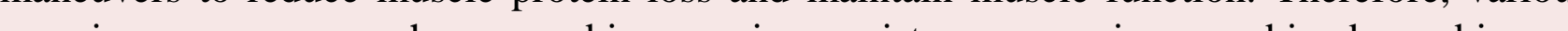
exerise prograse such as a for heme exterise and passive exercise including electrical stimulation have been develope

\section{Aim of the work}

The aim of this study was to compare the effect of intradialytic aerobic exercise alone versus combined aerobic and resistance exercise on arterial stiffness and the echocardiographic findings among hemodialysis patients.

\section{Patients and Methods}

PATIENTS: This study was conducted on 45 chronic kidney disease stage 5(CKD-5) patients receiving regular maintenance hemodialysis 4 hourly sessions 3 times per week Medical Research Institute Dialysis Unit.

The patients were randomly assigned into 3 groups:

Group (A): Aerobic exercise group, where 15 patients were assigned to a 30-minute aerobic exercise program per dialysis session, 3 times a week, for 3 months.

Group (B): Combined exercise group, where 15 patients were assigned to a combined

exercise program per dialysis session 3 times a week, for 3 month.

Croup (C): Control group, where 15 patients receiving regular maintenance hemodialysis were not assigned to any exercise program.

METHODS: Expeinent stoly with pre and post asessent after 12 weeks of post assessment after 12 weeks of Atradialycic exercise training program.

All paitents were subjected to the following:

Written informed consent.
- Complete physical examination. - Measurement of the body weight and height.

- Laboratory investigations: Calcium, Phosphorus and PTH

- Assessment of the hemodialysis dose: $\mathrm{eKt} / \mathrm{V}$ and urea reduction ratio (URR)

- Arterial stiffness assessment: Central BP, PP, augmentation index and pulse wave velocity were calculated with an ambulatory oscillometric device (Mobil-O-Graph).

- Echo-Doppler examination: Left ventricular dimensions and mass index, Left ventricular systolic and diastolic function and assessment of the right ventricular dimension and function.

Exercise programs: Group (A) performed twelve week intradialytic aerobic training three times per week during the first half of the HD session for thirty minutes using a cycle ergometer. Group (B) performed elbow flexion using a free weight dumbbell before aerobic exercise. Group (C) did not perform any exercise.

\section{Results}

Table 1: Comparison among the three studied groups according to Augmentation index

\begin{tabular}{|c|c|c|c|c|c|}
\hline Arterial stiffness & $\begin{array}{l}\text { Group A } \\
(\mathbf{n}=15)\end{array}$ & $\begin{array}{l}\text { Group B } \\
(n=15)\end{array}$ & $\begin{array}{l}\text { Group C } \\
(n=15)\end{array}$ & $\begin{array}{l}\text { Test of } \\
\text { sig. }\end{array}$ & $\mathbf{p}$ \\
\hline \multicolumn{6}{|c|}{ AIx (\%) } \\
\hline \multicolumn{6}{|l|}{ Pre exercise } \\
\hline Min. - Max. & $11.0-44.0$ & $5.0-40.0$ & $2.0-35.0$ & \multirow{3}{*}{$\mathrm{H}=4.905$} & \multirow{3}{*}{0.086} \\
\hline Mean \pm SD. & $25.67 \pm 8.04$ & $18.60 \pm 10.40$ & $20.73 \pm 11.06$ & & \\
\hline Median (IQR) & $25(20.50-28.50)$ & $15.0(11.50-21.0)$ & $21.0(11.50-31.0)$ & & \\
\hline \multicolumn{6}{|l|}{ Post exercise } \\
\hline Min. - Max. & $10.0-40.0$ & $4.80-38.0$ & $5.0-40.0$ & \multirow{3}{*}{$\mathrm{H}=5.777$} & \multirow{3}{*}{0.056} \\
\hline Mean \pm SD. & $25.25 \pm 7.96$ & $17.50 \pm 9.94$ & $23.23 \pm 11.94$ & & \\
\hline Median (IQR) & $\begin{array}{c}24.9(19.50- \\
28.5)\end{array}$ & $\begin{array}{c}14.80(10.90- \\
20.90)\end{array}$ & $\begin{array}{c}22.0(13.50- \\
35.50)\end{array}$ & & \\
\hline $\mathbf{Z}\left(\mathbf{p}_{0}\right)$ & $1.330(0.183)$ & $3.318^{*}\left(0.001^{*}\right)$ & $3.321^{*}\left(0.001^{*}\right)$ & & \\
\hline Decrease & $0.42 \pm 1.33$ & $1.10 \pm 1.33$ & \pm 2.38 & $\begin{array}{c}\mathrm{H}=28.140 \\
*\end{array}$ & $<0.001^{*}$ \\
\hline Sig. bet. grps & \multicolumn{3}{|c|}{$\mathrm{p}_{1}=0.154, \mathrm{p}_{2}<0.001^{*}, \mathrm{p}_{3}<0.001^{*}$} & & \\
\hline$\%$ of decrease & $1.91 \pm 4.40$ & $6.74 \pm 9.88$ & $-21.12 \pm 36.71$ & $\begin{array}{c}\mathrm{H}=29.946 \\
*\end{array}$ & $<0.001^{*}$ \\
\hline Sig. bet. grps & \multicolumn{3}{|c|}{$\mathrm{p}_{1}=0.095, \mathrm{p}_{2}<0.001^{*}, \mathrm{p}_{3}<0.001^{*}$} & & \\
\hline
\end{tabular}

$p_{1}: p$ value for comparing between group $\mathbf{A}$ and group $\mathbf{B}$

$p_{2}: p$ value for comparing between group $\mathbf{A}$ and group $\mathbf{C}$
$\mathrm{p}_{3}: \mathrm{p}$ value for comparing between group $\mathbf{B}$ and group $\mathbf{C}$

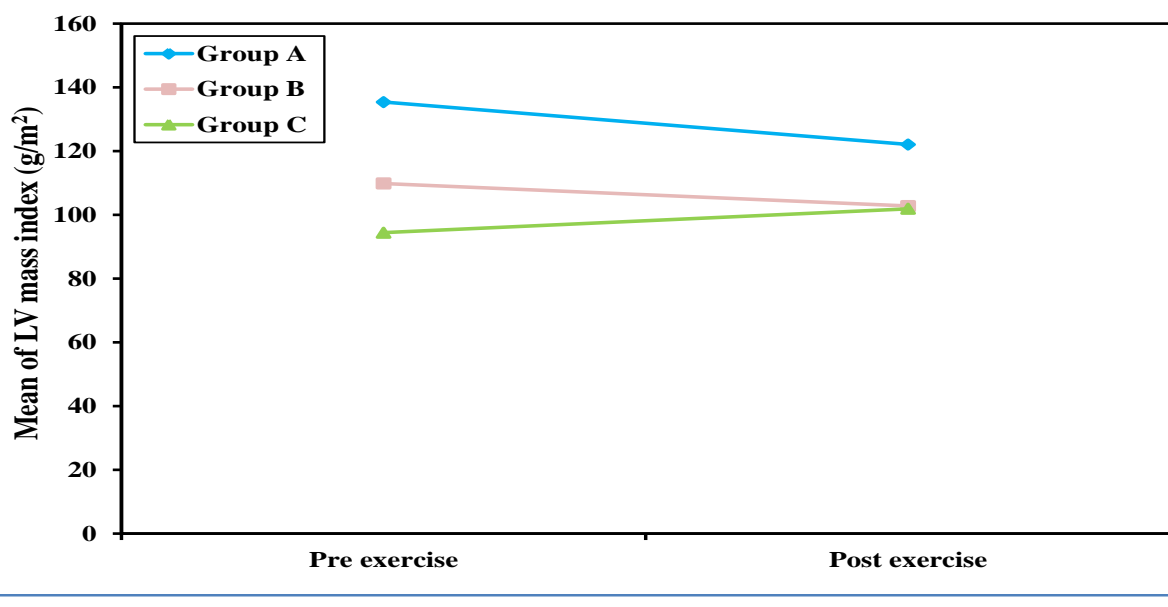

Figure 1: Comparison among the three studied groups according to LV mass index

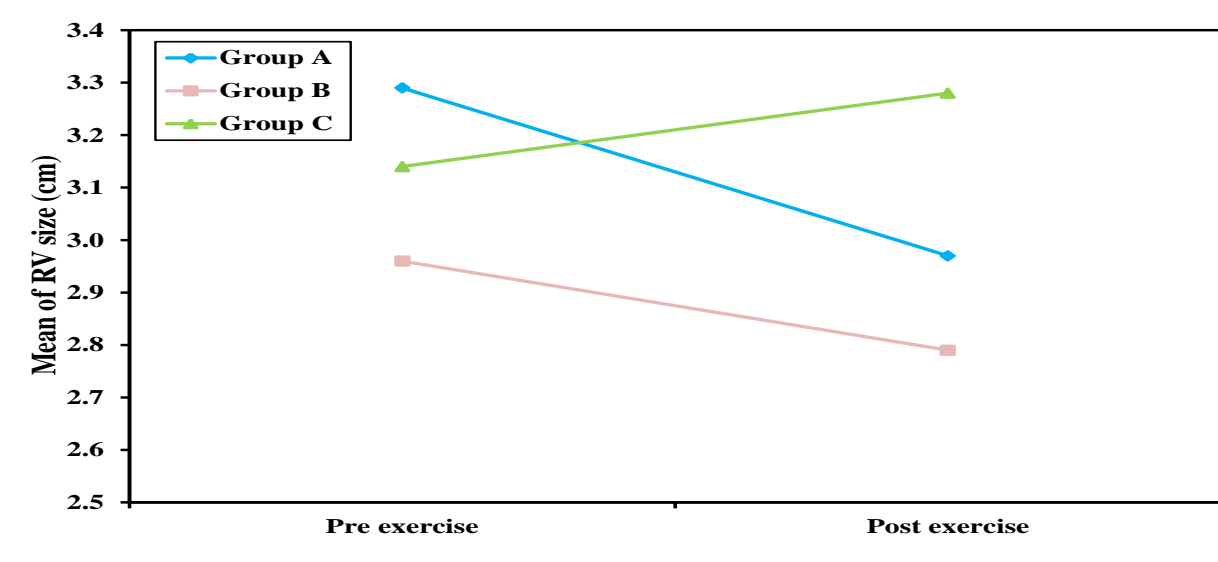

Figure 2: Comparison among the three studied groups according to right ventricular size

\section{Conclusion}

Cardiovascular comorbidities affects hemodialysis patients, so intradialytic exercise can have a powerful effect in improving dialysis efficacy expcially with combined exercise. Arterial stiffness shows marked

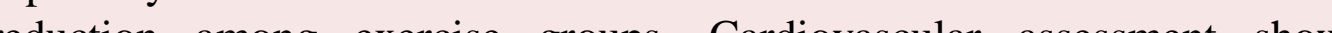
imp in HD who received exercise therapy.

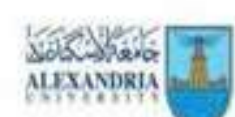

2021CAlexandria Faculty of Medicine MEDICINE |

$$
\text { CC-BY-NC }
$$

